\title{
Overweight or overfat? Many Canadians are both
}

- Cite as: CMAJ 2017 September 18;189:E1202-3. doi: 10.1503/cmaj.109-5472

Posted on cmajnews.com on Aug. 31, 2017.

$\mathrm{T}$ hree in five Canadians are overweight or obese, but a far greater number have potentially harmful levels of body fat, renewing debate about the best way to measure obesity.

According to Statistics Canada, $61.3 \%$ of adult Canadians were overweight or obese in 2015. The percentage of those who were obese rose to $26.7 \%$, up from $23.1 \%$ in 2004 . There was some good news when it came to younger Canadians: the percentage of children and youth who were obese dropped to $12 \%$ from $13.3 \%$.

The study measured obesity based on body mass index, or BMI, which is calculated using a person's height and weight. A provincial spokesperson for Manitoba (where child obesity is climbing) questioned the value of the measurement because "BMI does not consider lifestyle behaviours like dietary quality, physical activity, which are, in fact, stronger determinants of death and disease."

Leslie Geran of Statistics Canada acknowledged that BMI is an imperfect measure of obesity and might misclassify someone who is short and muscular as obese. But this doesn't explain higher rates in certain provinces, she said.

On the contrary, a recent study in Frontiers in Public Health suggests that using BMI to measure obesity likely underestimates the problem.

Researchers found that most adults in developed countries are "overfat" - that is, they have levels of excess body fat that put them at increased risk of a host of obesity-related diseases, including coronary heart disease, stroke, cancer and type 2 diabetes.

Even people considered "normal weight" can fall into this category, and commonly used BMI cutoffs to diagnose

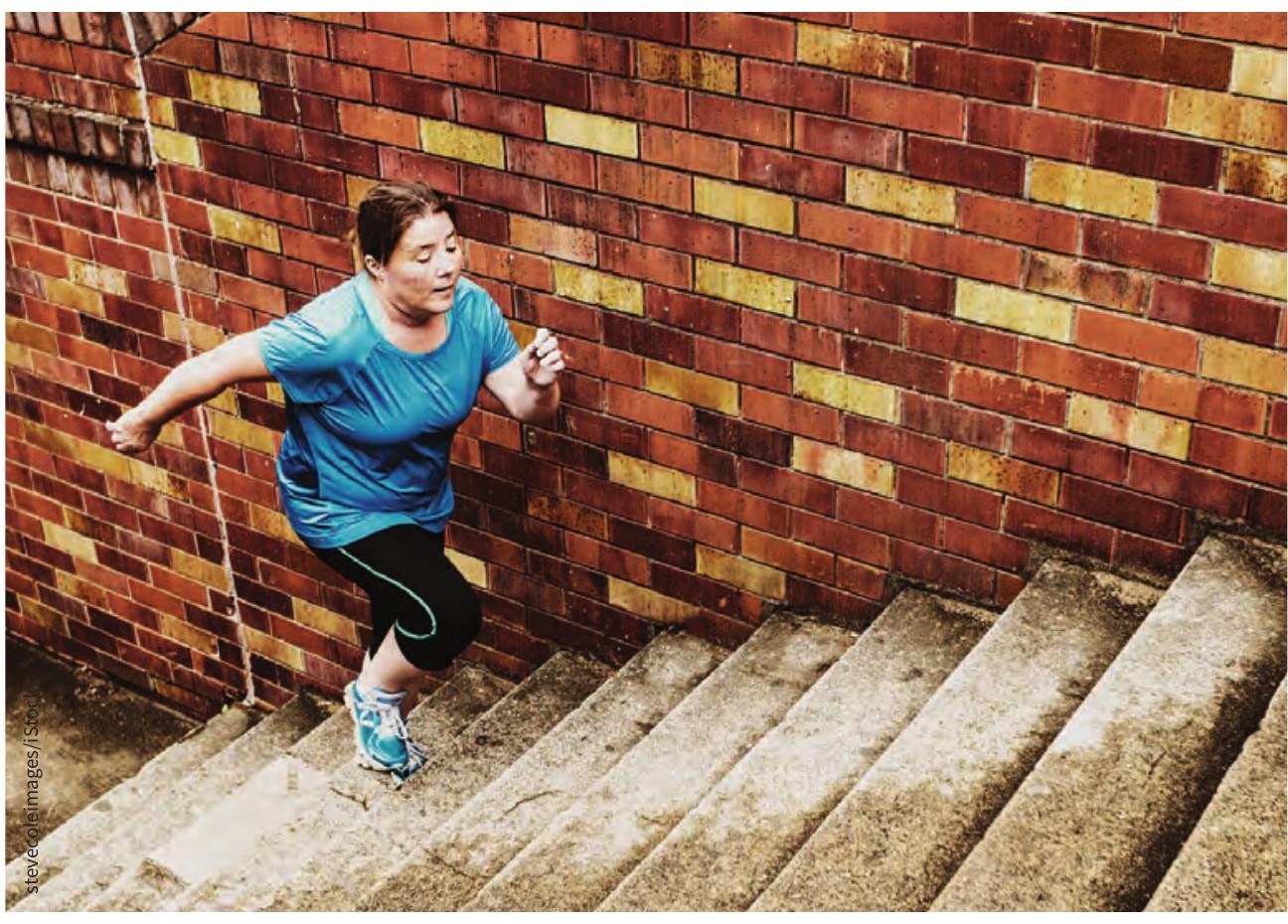

Focusing on body mass index misses the risks of high body fat in people of normal weight, researchers warn.

obesity miss about half of people with excess body fat.

"On average, the prevalence of overfat adults and children in developed countries is extremely high, and substantially greater than that of overweight and obese individuals," write Maffetone and colleagues in the Frontiers study. They found "alarmingly high" rates of overfat people in the United States and New Zealand, where more than $90 \%$ of men, $80 \%$ of women and $50 \%$ of children had unhealthy levels of body fat.

In Canada, an estimated $84.5 \%$ of men, $68.5 \%$ of women, $48.1 \%$ of boys and $44.2 \%$ of girls were overfat.

The authors note these rates are "substantially higher" than earlier estimates that up to $76 \%$ of the world's population has body fat levels that can impair health. This represents a "serious public health crisis" for developed countries, despite some reports that BMI-defined obesity is levelling off.

The "blanket adoption of BMI as the main indicator of obesity" has resulted in the "widespread, institutionalized underestimation" of unhealthy levels of body fat, they argue. "This has created a population of overfat individuals whose health needs may be unmet and overlooked."

Maffetone and colleagues propose measuring a person's waist instead of their weight to assess health risks. They attribute increasing rates of overfat "in great part to increased incidence of abdominal obesity," which has "separate and more severe health effects" than fat in other parts of the body. 
The researchers suggest the rule that a person's waist should be less than half their height. This waist-to-height ratio "may be the single best clinical indicator of health risk as it can be used throughout childhood, into adult life, as well as throughout the world."

The Canadian Task Force on Preventive Care guideline on managing obesity recommends waist circumference and waist-to-hip ratio as "acceptable mea- sures of obesity" alongside BMI, but doesn't provide specific instructions.

Dr. Robert Ross, a professor at Queen's University in Kingston, Ontario, told Global News such measures are more valuable than redefining obesity with terms like overfat. "They're making the argument we should use different terms, but I'm not sure I would." He suggested using both BMI and waist circumference measurements together.
Maffetone and coauthors argue in their study that "continued debate on how best to measure obesity and body fat may miss an important clinical point." Most clinicians "usually know if the patient in front of them has too much body fat," so they "should not have to wait for the latest consensus" to encourage lifestyle changes.

Lauren Vogel, CMAJ 